\title{
Impact of Cognitive Function Change on Mortality in Renal Transplant and End-Stage Renal Disease Patients
}

\author{
Akhil Sharma $^{\text {a }}$ Jonathan Yabes ${ }^{b}$ Saleem Al Mawed ${ }^{d}$ Christine Wu ${ }^{a}$ \\ Carol Stilley ${ }^{c}$ Mark Unruh $^{d}$ Manisha Jhamb ${ }^{\text {a }}$ \\ ${ }^{a}$ Renal-Electrolyte Division, Department of Medicine, ${ }^{b}$ Department of Medicine, and ${ }^{\mathrm{c} S}$ chool of Nursing and \\ Department of Psychiatry, University of Pittsburgh, Pittsburgh, Pa., ${ }^{d}$ Nephrology Division, Department of Internal \\ Medicine, University of New Mexico, Albuquerque, N.Mex., USA
}

\section{Key Words}

Kidney transplant $\cdot$ Cognitive function $\cdot$ Mortality

\begin{abstract}
Background: Limited evidence from small-scale studies, mainly involving end-stage renal disease (ESRD) patients, suggests that kidney transplantation may improve cognitive function. We examined changes in cognitive function after a kidney transplant and its association with survival in advanced chronic kidney disease (CKD)/ESRD patients. Methods: In a prospective study design, cognitive performance of 90 patients $(50.6 \pm 13.1$ years, $66.7 \%$ men, $27.8 \%$ blacks, $76 \%$ CKD stages 4-5) was assessed at the respective patients' residences using established neurocognitive tests. Results: Among the 90 patients, 44 received a kidney transplant (KTx group) while 46 did not (no-KTx group). After a mean follow-up of $\sim 19$ months, there was no significant change in scores for majority of cognitive tests in either group. Older age, but not diabetes or renal function status (CKD vs. ESRD), was a determinant of poor follow-up cognitive performance. Additionally, poor attention/psychomotor speed and executive performance (as measured by Trails A and Stroop test, respectively) was associated with higher mortality over a mean follow-up of 4.7 years, even after adjustment for age, sex, dia-
\end{abstract}

betes, CKD or ESRD status and kidney transplant status. Conclusion: Overall, cognitive function does not significantly improve after kidney transplant or significantly decline in non-transplanted, advanced CKD/ESRD patients. Poor attention, psychomotor speed and executive performance independent of transplant status were associated with higher mortality over time.

(c) 2016 S. Karger AG, Basel

\section{Introduction}

Up to $30-70 \%$ of end-stage renal disease (ESRD) and $17-30 \%$ of advanced chronic kidney disease (CKD) patients are reported to have significant cognitive deficits [1-4]. Cognitive impairment may affect the patients' daily functioning, independence, social adjustments, employment options, medication adherence, medical decision making [5] and is also an independent predictor of mortality $[6,7]$. Although there is a well-established survival benefit of renal transplantation, the evidence of its effect on cognitive function is limited and not entirely consistent.

Previous studies demonstrated that advanced CKD/ ESRD patients have significant impairments in verbal

\section{KARGER}

E-Mail karger@karger.com

www.karger.com/ajn
(C) 2016 S. Karger AG, Basel

0250-8095/16/0446-0462\$39.50/0
Assistant Prof. Manisha Jhamb, MD, MPH

Renal-Electrolyte Division, Department of Medicine University of Pittsburgh School of Medicine

200 Lothrop St., PUH C-1101, Pittsburgh, PA 15213 (USA)

E-Mail jhambm@upmc.edu 
memory and executive functioning $[1,2,4,8]$. There also appears to be an association between the severity of kidney disease and the degree of cognitive impairment [3, 5, 9]. The etiology of cognitive impairment in this population is multifactorial including high prevalence of cerebrovascular risk factors, cerebrovascular disease and increased exposure to acute changes in metabolic abnormalities/fluid shifts associated with renal replacement therapy (RRT) [10]. Kidney transplantation, perhaps by improving uremic milieu and metabolic abnormalities, may improve cognitive function. However, it is possible that the risk factors contributing to cognitive impairment cannot be corrected or reversed by transplant alone. In fact, cognitive function may worsen after the transplant as an after-effect of immunosuppressive medications.

Early studies demonstrated a beneficial effect of kidney transplantation on cognitive function, specifically on verbal memory [11-13]. More recent studies have revealed improvement in psychomotor speed, abstract thinking, attention and executive functioning with improvements sustaining well past the initial transplantation [14-16]. Although this is encouraging, these studies remain limited by small sample size, lack of non-transplant comparison group and lack of generalizability to the United States CKD/ESRD population. Moreover, almost all of these studies were performed only on patients undergoing hemodialysis (HD), and very limited information is available regarding the outcomes of non-dialysis CKD undergoing renal transplant.

The primary aim of our study was to examine the changes in cognitive function after a kidney transplant in a cohort of patients with advanced CKD/ESRD. We compared changes in cognitive function over time in patients who received a kidney transplant with those who did not. We hypothesized that those receiving the transplant will have improved cognitive function compared to those without a transplant. Finally, we examined whether baseline cognitive tests were associated with mortality in the transplanted $(\mathrm{KTx})$ and non-transplanted (no-KTx) groups.

\section{Subjects and Methods}

\section{Study Participants}

This cohort comprises data collected as part of a prospective study that investigated memory, sleep and quality of life in adult, English-speaking patients with advanced CKD (dialysis dependent or Modification of Diet in Renal Disease estimated glomerular filtration rate $(\mathrm{eGFR}) \leq 30 \mathrm{ml} / \mathrm{min} / 1.73 \mathrm{~m}^{2}$ ) or ESRD, defined as being on RRT (at least 3 times per week in-center HD

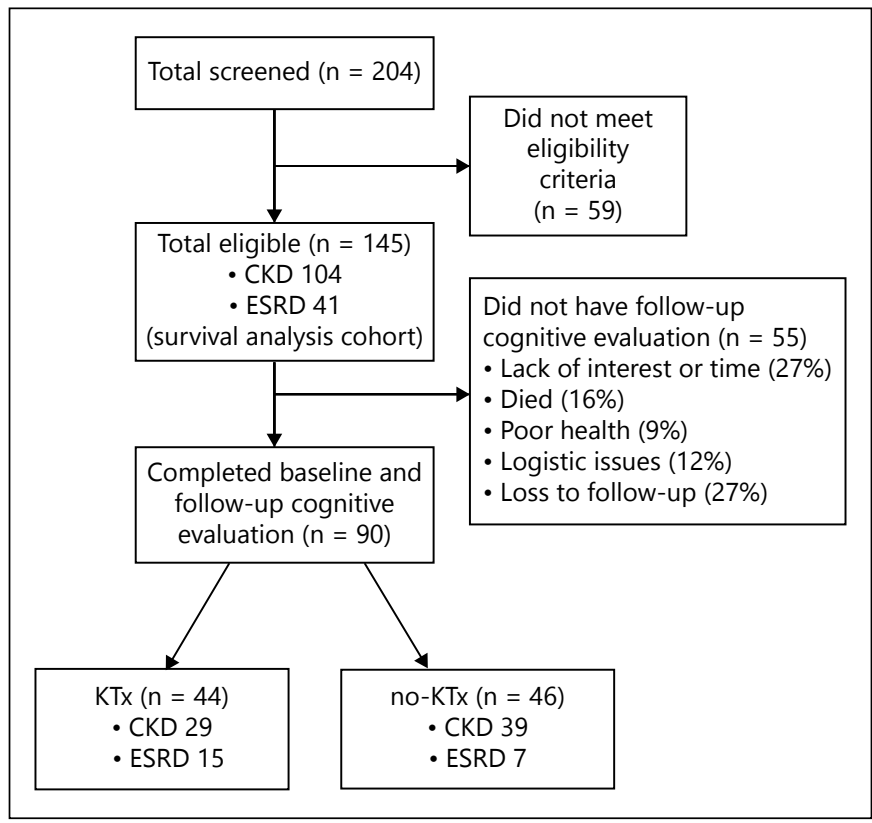

Fig. 1. Flowchart of patient selection for inclusion in the study cohort.

or peritoneal dialysis) for a minimum of 3 months [17]. We approached patients during routine $\mathrm{CKD}$ or dialysis clinic visit, or initial evaluation at a kidney transplant clinic in Western Pennsylvania between March 2004 and December 2008. Exclusion criteria included age $(<18$ or $>90$ years), craniofacial abnormalities, active malignancy, treated sleep apnea, active infection, active coronary artery disease, advanced cirrhosis, active alcohol abuse, history of head injury or refractory psychiatric disease. Patients with severe cognitive impairment (advanced dementia, clinically confused or those unable to provide basic personal information) were also excluded. Baseline cognitive evaluation was performed shortly after enrollment during initial home visit and patients were followed up prospectively. All participants signed informed consent and the study was approved by the University of Pittsburgh Institutional Review Board (PRO14010028).

Out of the 145 patients who met the eligibility criteria and consented to this study, 55 failed to complete follow-up cognitive evaluation (fig. 1). Among the 90 participants who completed a baseline and follow-up cognitive evaluation per study protocol, 44 (15 ESRD and $29 \mathrm{CKD}$ ) received a transplantation during study period (KTx group) and completed follow-up cognitive evaluation at least 6 months after transplant. We chose this interval to allow for stabilization of renal allograft function and immunosuppressive medication regimen. The remaining 46 patients (7 ESRD and $39 \mathrm{CKD}$ ) did not receive transplantations during study period (noKTx group; fig. 1).

\section{Data Collection}

Baseline data were collected from a standardized health interview and questionnaire that included the assessment of previous medical problems, surgeries and relevant medical procedures. Current medications and anthropometric measurements were ob- 
Table 1. Description of cognitive tests used

\begin{tabular}{|c|c|c|}
\hline Cognitive test & Brief description & Measurement criteria \\
\hline CLOX 1 and $2[39,40]$ & $\begin{array}{l}\text { Assesses abstract thinking and visual spatial constructive } \\
\text { abilities }[39,40] \text {. }\end{array}$ & $\begin{array}{l}\text { Scored based on accuracy. } \\
\text { Higher score is better }\end{array}$ \\
\hline $\begin{array}{l}\text { Controlled Oral Word } \\
\text { Association (COWA) [41] }\end{array}$ & $\begin{array}{l}\text { Language assessment test for phonetic/semantic fluency } \\
\text { components. Individual required to produce words with a } \\
\text { designated first letter and a list of animals each within a } 1 \mathrm{~min} \\
\text { period [41] }\end{array}$ & $\begin{array}{l}\text { Combined score used. Higher } \\
\text { score is better; } 20 \text { words or less } \\
\text { is considered seriously } \\
\text { deficient }\end{array}$ \\
\hline Digit Span Subtest [42] & $\begin{array}{l}\text { Individual is required to repeat increasingly longer number } \\
\text { sequences in either forward or reverse order. A subtest from } \\
\text { Wechsler Memory Scale [42] }\end{array}$ & $\begin{array}{l}\text { Age-based scaled score } \\
\text { generated and used }\end{array}$ \\
\hline DSST [42] & $\begin{array}{l}\text { Test for visual scanning and rapid response where individual } \\
\text { substitutes numbers for symbols based on a learned code. } \\
\text { A subtest from the Wechsler Memory Scale [42] }\end{array}$ & $\begin{array}{l}\text { Score equaled total number } \\
\text { correct within the given time } \\
\text { frame }\end{array}$ \\
\hline Logical Memory Subtest [42] & $\begin{array}{l}\text { Assessment for verbal memory. Individual is read a story, asked to } \\
\text { recall story related details immediately and } 30 \text { min post. A subtest of } \\
\text { Wechsler Memory Scale [42] }\end{array}$ & $\begin{array}{l}\text { Age-based standard score was } \\
\text { used }\end{array}$ \\
\hline MMSE $[43,44]$ & $\begin{array}{l}\text { Tests orientation, attention, calculation, recall, language, and visual } \\
\text { spatial skills }[43,44]\end{array}$ & Raw scores used \\
\hline $\begin{array}{l}\text { RCF Copy Condition } \\
\text { test }[18,45]\end{array}$ & $\begin{array}{l}\text { Assesses visual spatial/constructional abilities. Requires individuals } \\
\text { to copy complex figures with immediate spontaneous and delayed } \\
\text { (30 min later) recall trial }[18,45]\end{array}$ & $\begin{array}{l}\text { Each copy is scored for } \\
\text { accurate reproduction and } \\
\text { placement of specific elements } \\
\text { of figure. Higher score is better }\end{array}$ \\
\hline WCST [47] & $\begin{array}{l}\text { Assessment for problem solving that requires flexibility in the face of } \\
\text { changing schedules of reinforcement. Individual is given a set of } \\
\text { stimulus cards, instructed to match the cards but not told how, and } \\
\text { then told whether correct or not [47].Computerized version used }\end{array}$ & $\begin{array}{l}\text { Number of completed } \\
\text { categories reported. Higher } \\
\text { score is better }\end{array}$ \\
\hline
\end{tabular}

tained using data regarding the self-reported age, race and socioeconomic status. Baseline serum laboratory tests were collected from medical record.

\section{Neurocognitive Assessment}

Patients were assessed using a battery of well-validated neurocognitive tests with high inter- and intra-rater reliabilities (table 1). Tests were administered by research assistants trained and supervised by an expert in neuropsychological assessment (C.S.). To ensure a quiet, distraction-free environment, assessments were performed at the patients' residences. By avoiding assessment during HD, we minimized the effect of rapid fluid/electrolyte shifts during HD on cognitive performance. The tests were administered in a standardized way using the same sequence of tests and on paper except the Wisconsin Card Sorting Test (WCST), which was administered on a laptop.

\section{Definition of Exposures}

Depression was defined as Patient Health Questionnaire-9 score of $\geq 10$, with 5 or more symptoms - including either depressed mood or anhedonia - present for more than half the day 
over the prior 2 weeks and/or self-reported treatment with anti-depressants. Diabetes was evaluated by both self-report and/or current use of insulin or hypoglycemic agents. Cardiovascular disease was evaluated by patient self-reporting (of previous myocardial infarction or heart attack, stroke or previous coronary artery bypass/angioplasty). Patient's overall health-related quality of life was assessed using the short-form health survey (SF-36).

\section{Statistical Analysis}

Categorical and continuous variables were presented using frequencies with percentages and means with SDs (or medians with IQR for skewed distributions), respectively. Baseline characteristics were compared between KTx and no-KTx groups. Baseline, follow-up and changes in neurocognitive measures were also compared between the 2 groups. Within-group changes in neurocognitive measures from baseline to follow-up were assessed using linear mixed model with random subject intercept. Between-group comparisons used two-sample $t$ test or Wilcoxon rank sum test and chi-square or Fisher exact test for continuous and categorical measures, respectively. Adjusted analyses of change scores of each neurocognitive measure were conducted using linear models that were adjusted for age, gender, baseline cognitive score and intelligence quotient (IQ). IQ score was used as a proxy for education due to limited capture of the education data by the binary "high school graduate' variable. In multivariable linear regression models stratified by KTx group, we examined the association between select follow-up neurocognitive tests (Mini Mental Status Examination (MMSE), Logical Memory 1 and 2, and Trails A and B) and patient characteristics (age, diabetes, renal function status (CKD vs. ESRD) and IQ score) that may be important determinants of cognitive function after adjusting for the baseline cognitive score. Due to skew, Trails A and B were log-transformed prior to modeling. Within the KTx group, pre- and post-transplant neurocognitive scores were compared between CKD and RRT using 2-sample t test or Wilcoxon rank sum test.

To examine the relationship between cognitive performance (MMSE, Logical Memory 1 and 2, Trails A and B) and overall survival, we fitted Cox proportional hazards model with and without adjusting for age, gender, diabetes, renal function status and a time-dependent covariate, receipt of kidney transplant. Hazards ratio (HR) along with the 95\% CIs were reported. National Death Index data until December 31, 2011, were obtained and patients still alive on this date were censored.

For sensitivity analysis, we applied principal component (PC) analysis (PCA) of the neurocognitive measures to reduce the dimensionality. We examined the scree plot, Eigen values and $\mathrm{cu}-$ mulative proportion of variance to determine the number of PCs to keep. Loadings and contributions of the neurocognitive measures were used to characterize the PCs and to examine agreement with the components of commonly presented latent constructs. PC scores were calculated and analyzed using the same methods applied to the original neurocognitive measures. The tests included in PCA analysis were Clock Drawing Test (CLOX 1 and 2), Controlled Oral Word Association, Logical Memory 1 and 2, Trails A and B, Stroop, Digit Span Forward and Backward, Digit Symbol Substitution Subtest (DSST), Rey-Osterrieth Complex Figure (RCF) Copy Condition test and WCST.

All statistical analyses were carried out in $\mathrm{R}$ (version 13.2) [18] using the dplyr package [19] for data manipulation, group comparisons [20] for descriptive tables, ggplot2 [21] for graphics, survival [22] for survival analysis and lme4 for linear mixed modeling.

\section{Results}

Baseline characteristics of the KTx and no-KTx group are shown in table 2. Patients who received KTx had significantly lower prevalence of diabetes (27.3 vs. $53.3 \%$, $\mathrm{p}=0.02$ ) as compared to the no-KTx group. The KTx group had trends toward more patients on RRT and lower eGFR among non-dialysis-dependent CKD (eGFR $16.2 \pm 4.9$ vs. $19.9 \pm 8.0, \mathrm{p}=0.04)$ than the no-KTx group. Except for better self-reported physical health (SF-36 PCS) in the KTx group, both groups were similar in terms of baseline demographics, comorbidities (including history of stroke), cognitive reserve (IQ score), mental health including depression, antidepressant or antihistamine use and biochemical status. Among the KTx group, those with non-dialysis-dependent CKD were more likely to be white, employed, less anemic and have higher cognitive reserve (IQ score) and lower SF-36 mental component summary score as compared to those with ESRD (online suppl. table 1; for all online suppl. material, see www. karger.com/doi/10.1159/000451059). Among the noKTx group, there were no differences in any demographics, comorbidities or biochemical variables (online suppl. table 2). At CE\#1, the KTx and no-KTx group had similar scores for all cognitive tests except CLOX 1 and 2, for which the KTx group performed slightly better (table 3 ).

In our KTx group, $78 \%$ of patients were on a single immunosuppressant, most commonly tacrolimus (95\% patients), with an average daily dose of $8.1 \mathrm{mg}$. CellCept was used in $22 \%$, Rapamune in $4.9 \%$ and a combination of one of these with steroids in $2.4 \%$ of the patients. The average daily doses of CellCept, Rapamune and steroids were 984, 4 and $30 \mathrm{mg}$, respectively.

\section{Longitudinal Change in Cognitive Function}

The time interval between the first (CE\#1) and the final ( $\mathrm{CE} \# 2$ ) cognitive evaluation for the entire cohort was $19.1 \pm 9.0$ months and was comparable between the KTx and no-KTx groups (table 1). For KTx group, the mean time interval from kidney transplant to CE \#2 was $8.3 \pm 5.2$ months. Table 4 shows the longitudinal changes in cognitive scores for both the groups. In the KTx group, there was no significant change in cognitive scores for all tests except slight improvement in RCF delayed recall testing $(19.1 \pm 8.4$ vs. $17.0 \pm 7.8, \mathrm{p}=0.02)$ compared to 
Table 2. Baseline characteristics comparing KTx vs. no-KTx group

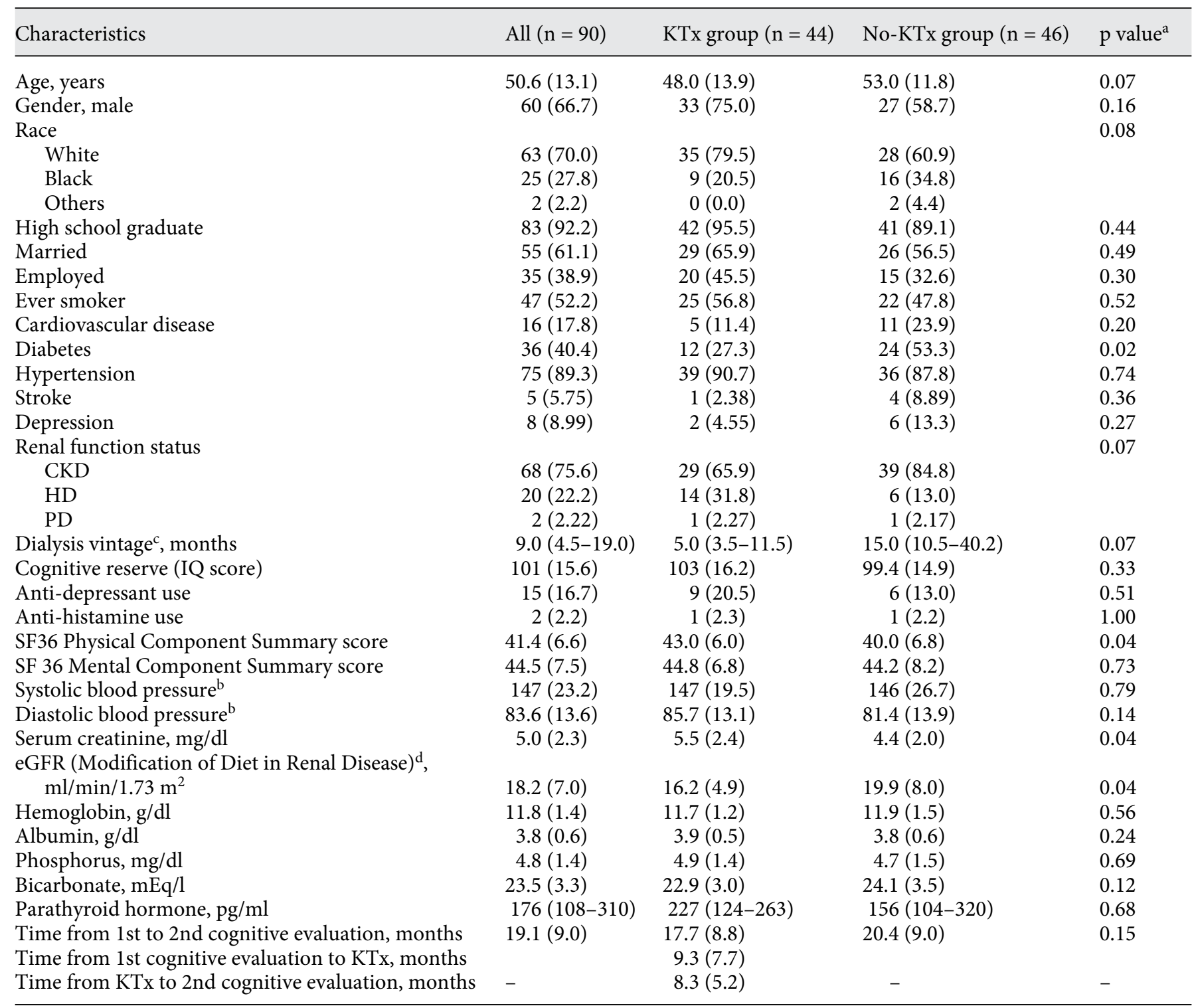

Data are presented as percentage $\mathrm{n}(\%)$, mean (SD) or median (Q1-Q3) where Q1 is the 25th percentile and Q3 is the 75th percentile. ${ }^{a} \mathrm{p}$ values use analysis of variance or Kruskal-Wallis for continuous variables and Fisher exact or chi-square for categorical variables.

$\mathrm{b}$ Blood pressure was measured during home visit at the time of cognitive evaluation.

${ }^{\mathrm{c}}$ Dialysis vintage missing for 1 patient.

${ }^{\mathrm{d}}$ For non-dialysis-dependent CKD only.

pre-transplant score. We also tested whether the patients' post-transplant scores were different based on patients' CKD or ERSD status at baseline and found no significant difference (online suppl. table 3). Similarly, in the no-KTx group, there was no change in cognitive scores over a period of $20.4 \pm 9.0$ months. Figure 2 displays unadjusted and adjusted (for age, sex, baseline IQ (as proxy for edu- cation) and baseline cognitive score) mean difference of longitudinal changes in cognitive scores between KTx and no-KTx groups. Overall, there was no significant difference in the change of cognitive function over time in the 2 groups except for greater improvement in Logical Memory 2 within KTx group (adjusted HR 1.08 (95\% CI $0.20-1.96) ; \mathrm{p}=0.02$ ). 
Table 3. Baseline cognitive scores (CE\#1) comparing KTx vs. no-KTx group

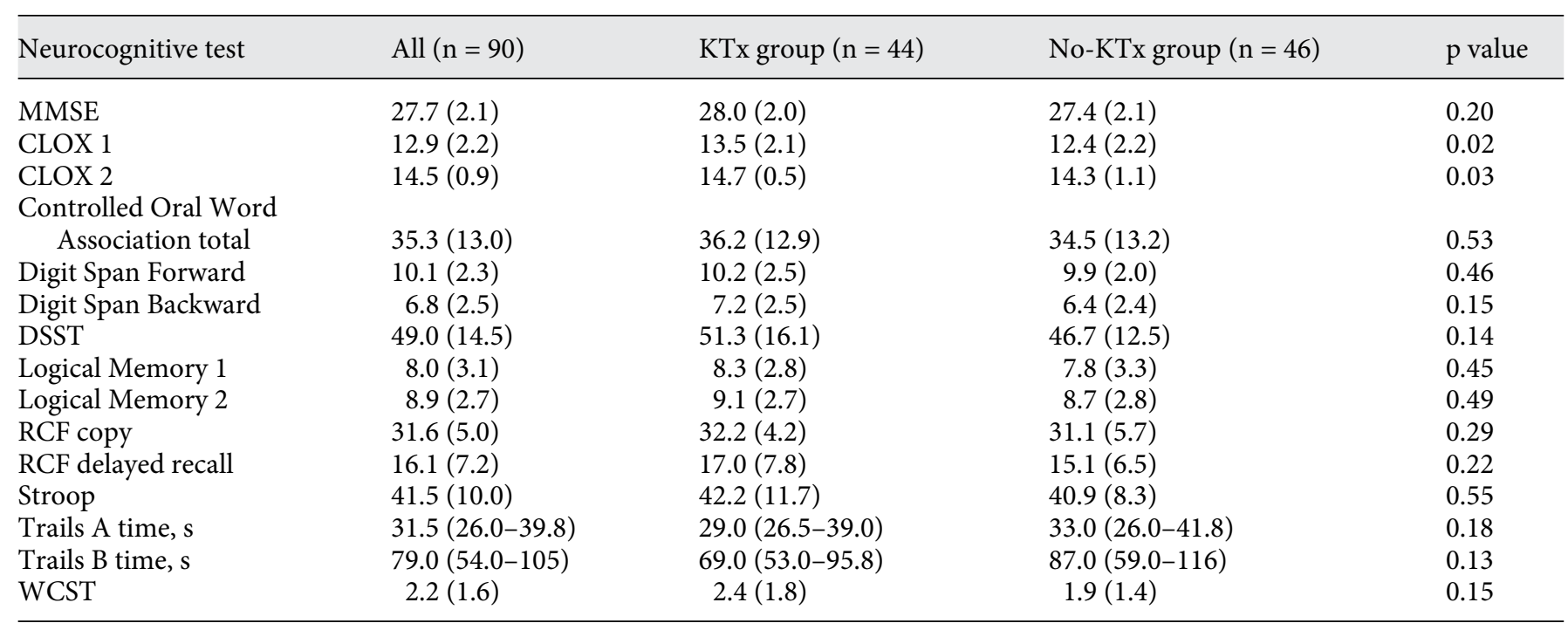

Data are presented as percentage $\mathrm{n}(\%)$, mean (SD) or median (Q1-Q3) where Q1 is the 25th percentile and Q3 is the 75th percentile.

\section{Determinants of Cognitive Function}

We conducted multivariable analysis of association of CE\#2 with select patient characteristics that may be important determinants of cognition (age, baseline IQ (as proxy for education), diabetes, renal function status (CKD vs. ESRD)) after adjusting for baseline score and stratification by transplant status. For both the KTx and no-KTx groups, lower IQ was associated with lower MMSE score but did not associate with Trails A or B or Logical Memory 1 or 2 . Within the no-KTx group, increasing age was associated with poorer performance on MMSE, Trails A, Logical Memory 1 and 2. Diabetes and renal function status were not significant determinants of cognitive function for either of the group (online suppl. fig. 1).

\section{Association of Cognitive Function with Survival}

For survival analysis, our cohort included all subjects who had completed a baseline cognitive evaluation ( $\mathrm{n}=$ 145). There were 35 deaths over a mean follow-up period of $4.7 \pm 1.8$ years. Association of baseline cognitive scores with mortality was assessed by adjusting for transplant status as a time-dependent variable. In unadjusted analysis, poor performances on Trails A, Trails B, DSST and Stroop test were associated with higher mortality (unadjusted HR 3.03 (95\% CI 1.53-6.02); $\mathrm{p}=0.002$ for Trails A; 3.25 (95\% CI 1.60-6.61); $\mathrm{p}=0.002$ for Trails B, 0.97 (95\% CI $0.94-0.99) ; \mathrm{p}=0.016$ for DSST; 0.94 (95\% CI $0.90-$
0.98); $\mathrm{p}=0.002$ for Stroop; fig. 3). However, after adjustment for age, sex, diabetes, CKD or ESRD status and kidney transplant status, only poor performance on Trails A and Stroop were significantly associated with mortality (adjusted HR 3.47 (95\% CI 1.19-10.1); $\mathrm{p}=0.02$ and 0.93 (95\% CI 0.88-1.00); $\mathrm{p}=0.04$, respectively). There was no association of baseline MMSE, CLOX 1 or 2, Logical Memory 1 or 2, RCF copy, RCF delayed recall, Digit Span Forward or Backward with mortality.

\section{Sensitivity Analysis Using PCA}

We employed PCA to derive a composite score of cognition. The number of PCs to keep, however, was not consistent among the different criteria. The scree plot showed that 1 component is sufficient, but only $36 \%$ of the variance would be accounted for. The criterion of Eigen value $>1$ would keep 5 components, which would account for about $70 \%$ of the variance. We would have to keep $7 \mathrm{com}-$ ponents in order to account for at least $80 \%$ of the variability. These findings suggest that PCA was not very effective for this data set. Nevertheless, we chose to keep 5 components. Based on the neurocognitive loadings and contributions, these components represent (1) executive functioning, (2) logical memory, (3) digit span, (4) RCF and (5) CLOX. There was no significant difference between the KTx and no-KTx groups' baseline or follow-up cognitive functioning. In the KTx group, the only significant change was a slight improvement in CLOX (post-pre 


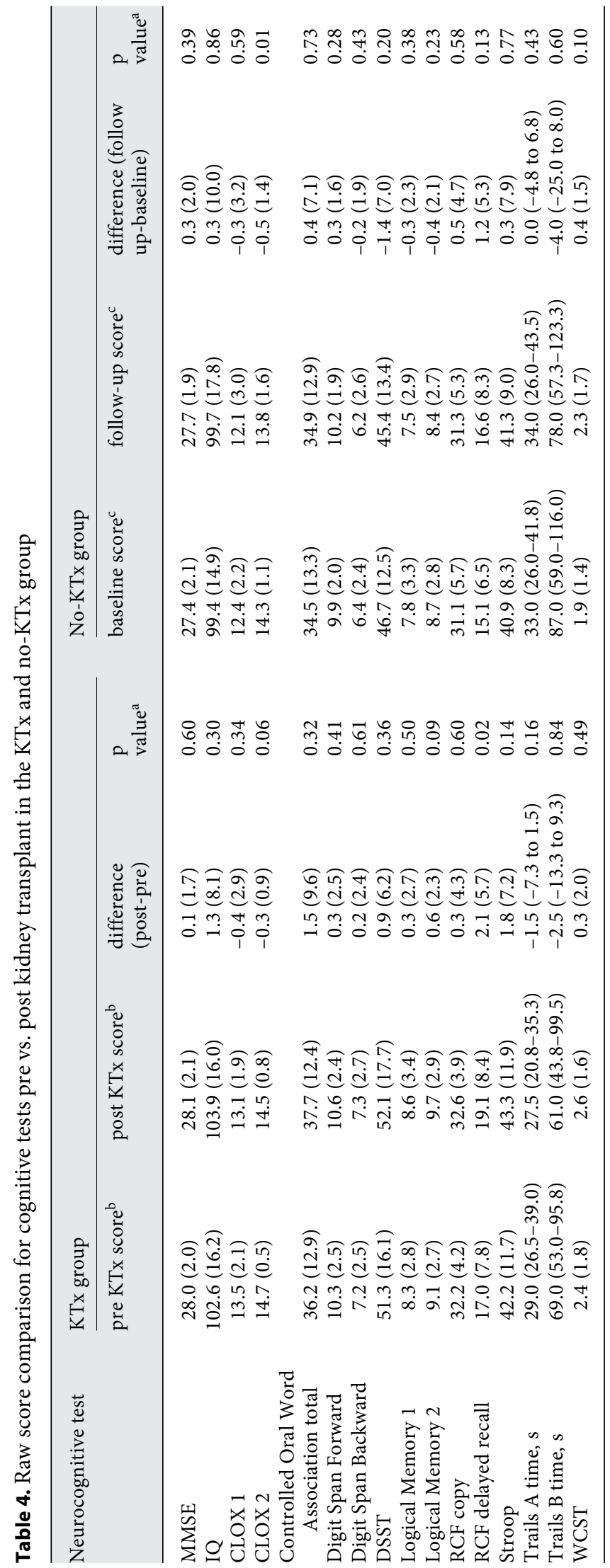

Tx $0.3(1.0) ; \mathrm{p}=0.04)$. For survival analysis using PCA, we found that worser performance on PC Executive functioning was associated with mortality in unadjusted analysis (HR 0.82 (95\% CI 0.68-0.99); $\mathrm{p}=0.04$ ); however, this became insignificant when adjusted for age, sex, diabetes, CKD or ESRD status and KTx status.

\section{Selection Bias Analysis}

To rule out the selection bias, we compared the characteristics of the study cohort for patients who had 2 cognitive evaluations $(\mathrm{n}=90)$ with patients who completed only baseline cognitive evaluation $(n=55)$ and subsequently dropped out. The groups were similar in terms of demographics, comorbidities and renal function status, with the only significant difference being dialysis vintage duration (median 9.0 (4.5-19.0) months for study cohort vs. 45.0 (11.0-61.0) months, $\mathrm{p}=0.01$ for the drop-outs; online suppl. table 4). There was no significant difference in any of the baseline cognitive scores among the 2 groups (online suppl. table 5).

\section{Discussion}

Our prospective study demonstrated that kidney transplantation was not associated with significant improvement in cognitive function among patients with advanced $C K D / E S R D$. In patients with advanced $C K D /$ ESRD who had not received a transplant, cognitive function remained stable over a mean follow-up of about 20 months. Multivariable stratified analysis demonstrated that diabetes and renal function status (CKD vs. ESRD) were not associated with changes in cognitive performance over time, but lower age was associated with better performance on certain tests. We also found that poor attention/psychomotor speed and executive performance (as measured by Trails A and Stroop test, respectively) were significantly associated with higher mortality over a

(footnote to table 4)

Data are presented as mean (SD) or median (Q1-Q3) where Q1 is the 25th percentile and Q3 is the 75th percentile.

${ }^{a} \mathrm{p}$ value obtained from unadjusted random intercept model.

b In KTx group, for baseline (pre KTx score)-Stroop and WCST missing for 2 subjects. For post-KTx score, CLOX-1 and CLOX-2 scores missing for 1 subject and WCST missing for 3 subjects.

${ }^{c}$ In no-KTx group, for baseline scores - Trail B missing for 1 subject, WCST missing for 2 subjects, Stroop missing for 1 subject. For followup scores, RCF copy missing for 6 subjects, RCF delayed recall missing for 1 subject, WCST missing for 2 subjects, Stroop missing for 3 subjects. 
Fig. 2. Mean difference in the longitudinal change in cognitive scores (post-pre) in KTx vs. no-KTx group. Adjusted for age, sex and baseline cognitive score. ${ }^{*}$ Effect per 5 unit increase.

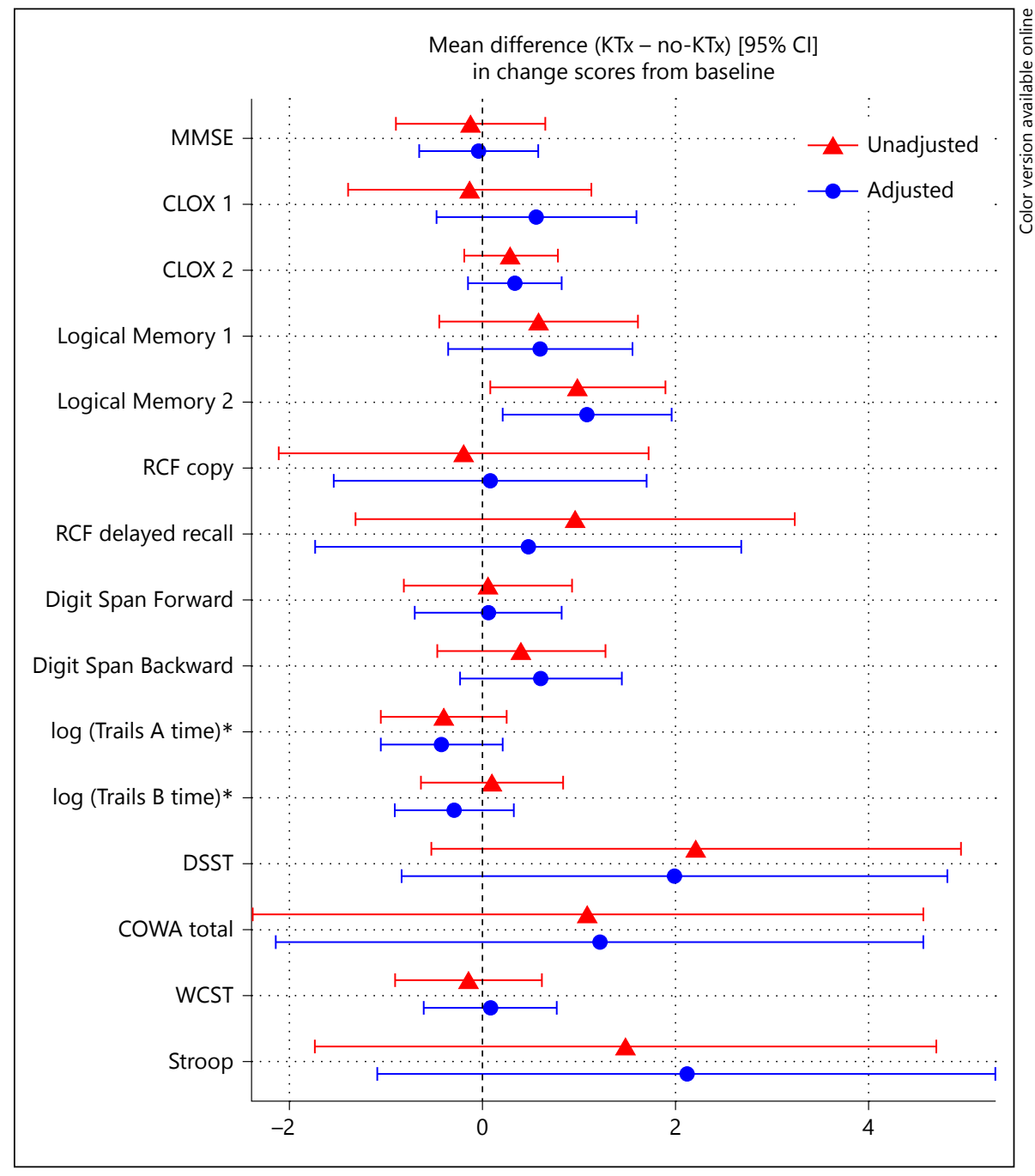

mean follow-up of 4.7 years, even after adjustment for age, sex, diabetes, CKD or ESRD status and kidney transplant status.

Our study adds to the existing literature by examining the effect of transplant on cognitive function in a representative US CKD/ESRD population. While most studies have evaluated the effect of transplant only in HD patients, we extend these findings to non-dialysis-dependent CKD patients, which comprised $76 \%$ of our cohort. A unique strength of our study was that both baseline and follow-up cognitive assessments were performed in the patients' residences, away from distractions and acute effect of HD, as opposed to during HD or in a research office in prior studies, which by itself may have introduced bias [13-16]. There is emerging evidence that, in addition to the uremic milieu, HD procedure it- self poses significant circulatory stress and causes irreversible ischemic brain injury in this population that is having already-existing vascular diseases [23]. The longer dialysis vintage in our cohort compared to most other studies (median 5 years compared to mean $2.9 \pm 2.5$ years in Gelb et al., $2.6 \pm 2.7$ years in Griva et al. [13] and $3.6 \pm 3.6$ years in Harciarek et al. [14]) may have contributed to lack of improvement in cognitive performance in our study [24]. Another possible explanation may be the patient characteristics of our cohort, which was slightly older than in most other studies and may have chronic aging-related changes in cerebrovascular structure/function that are not reversed by transplantation. We did find that older age was strongly associated with diminished cognitive performance. Our patients also had a higher prevalence of diabetes, which is a risk factor for micro- 
Fig. 3. Association of baseline cognitive function with survival. * HR per 0.25 unit increse.

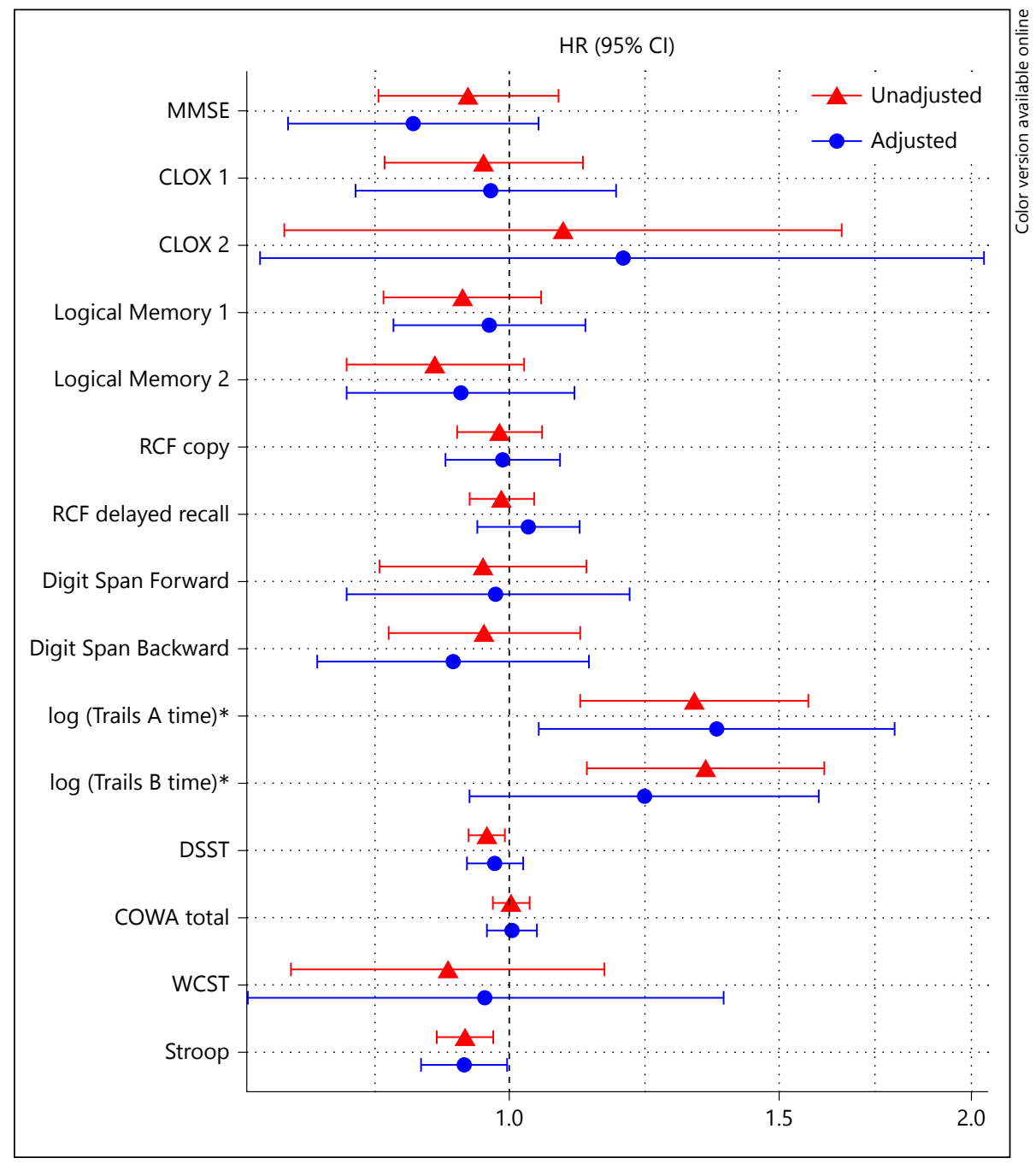

vascular changes, although we did not see any differences in our stratified analysis, which was underpowered. Although the KTx group had more ESRD patients (34\%) than the no-KTx group (15\%), we do not feel that this affected our results. It may be argued that cognitive function may be directly correlated with the severity of kidney disease. However, the baseline cognitive scores for the KTx and no-KTx groups were similar for all cognitive tests except CLOX 1 and 2, for which the KTx had slightly better scores. Moreover, there was no significant difference in post-transplant scores for any of the tests based on the patients' pre-transplant renal function status or association of CE\#2 with renal function status in multivariable analysis.

The high prevalence of traditional vascular risk factors, such as hypertension and diabetes in CKD/ESRD patients, may contribute to the high prevalence of clinical and subclinical cerebrovascular disease leading to abnormalities such as cerebral atrophy, silent brain infarcts and white matter hyper intensities [25-29]. Studies indicate that cognitively impaired ESRD patients have more frequent and severe multiple infarct dementia, vascular dementia and cerebrovascular lesions even in the absence of clinical stroke [30-33]. Such findings suggest that cerebrovascular disease is the primary cause of cognitive impairment in this population [23], thus not likely to be corrected or reversed by kidney transplant.

An interesting finding in our study was stable cognitive function in no-KTx advanced CKD/ESRD patients over time, contrary to some studies suggesting decline in cognition in ESRD patients over a period of 2 years [15]. It may be that cognitive function is better preserved over time in non-dialysis CKD patients, which was the majority of our cohort. Additionally, the mean 
baseline IQ our cohort was $101 \pm 15.6$ (near average per normative data), which may have contributed to better cognitive preservation or test performance over time. Alternatively, we might have selected a group of patients who are more cognitively intact, although we did not find any evidence of selection bias when compared to patients who had completed only baseline evaluation.

To our knowledge, none of the prior studies assessing cognitive function and mortality in CKD accounted for transplant status [27]. We found that poor baseline cognitive function, especially attention, psychomotor speed and executive functioning was independently associated with higher mortality. Despite reaching statistical significance, our results should be interpreted cautiously given the limited sample size and multiple testing in our study. Nevertheless, our findings are consistent with and add to prior literature, and may have important clinical implications in identifying high-risk patients [7].

Our study has several areas of strength. Ours is the largest study evaluating the effect of kidney transplant using a prospective study design. Moreover, the inclusion of a CKD/ESRD control group of patients who did not receive kidney transplant allows for better comparison between the KTx and no-KTx groups and assessment of longitudinal changes in the no-KTx group over time. Additionally, unlike most prior studies, we administered in-home cognitive tests both times, thus minimizing the effect of rapid fluid/electrolyte shifts and hemodynamic changes during HD on cognitive function as well providing a consistent testing environment across the 2 tests. Lastly, we accounted for depression and medications that may impair cognition, both of which may be important confounders in this patient population [34].

Our study has some limitations. We did not have longer term cognitive follow-up to evaluate the effect of transplant on cognition. However, the mean time from kidney transplant to follow-up evaluation in our study was $8.5 \pm 5.3$ months, which should have allowed adequate time for stabilization of renal function and immunosuppressive drug levels after the transplant. Moreover, prior studies have demonstrated changes in cognitive function over a similar follow-up time interval $[13,14$, 35]. Thus, longer follow-up would not be expected to change our results. Second, although a large number of patients who initially enrolled in the study failed to complete follow-up testing, we did not find any evidence of selection bias in terms of demographics, comorbidities, renal function status or baseline cognitive function. How-

Pre- and Post-Transplant Cognitive Function ever, it is possible that there may be differences in other variables that were not captured in our study, thus limiting the generalizability of our findings Third, the transplant allocation was non-random, and one could posit that those who had received a transplant had higher baseline and follow-up cognitive performance and may be more likely to have a ceiling effect. However, we did not find any significant differences in baseline cognitive scores or follow-up scores for any of the measured domains in our cohort. Fourth, although we did not adjust for the effect of immunosuppressive medications on cognitive performance, a contemporary lower immunosuppression protocol was the approach at our transplant center during the time period of this study. Lastly, because of the small number of deaths in our cohort, especially in the KTx group, we were not able to adjust for variables that may affect patient survival.

In conclusion, in a prospective study of CKD/ESRD patients receiving a kidney transplant compared with those who did not receive a transplant, we observed no significant improvement in cognitive function post the transplant. Moreover, we did not find any significant decline in cognitive function over time in those CKD/ESRD patients who did not receive a transplant. We did find poor that cognitive function, especially attention, psychomotor speed and executive functioning, was independently associated with increased mortality. More studies are needed to investigate the determinants of cognitive function in this population and long-term effects on cognitive function post the kidney transplant.

\section{Acknowledgment}

We would like to thank Mary Fletcher, BS, for help with all data management.

\section{Sources of Support}

This work was supported by National Institute of Diabetes and Digestive and Kidney Diseases grant P30-DK-079307, R01DK077785 (M.U.) and American Heart Association grant 11FTF7520014 (M.J.). The content is solely the responsibility of the authors and does not necessarily represent the official views of the National Institutes of Health.

\section{Disclosure Statement}

The authors of this manuscript have no conflicts of interest to disclose. 


\section{References}

1 Sarnak MJ, Tighiouart H, Scott TM, et al: Frequency of and risk factors for poor cognitive performance in hemodialysis patients. Neurology 2013;80:471-480.

2 Murray AM, Tupper DE, Knopman DS, et al: Cognitive impairment in hemodialysis patients is common. Neurology 2006;67:216223.

3 Kurella M, Chertow GM, Luan J, Yaffe K: Cognitive impairment in chronic kidney disease. J Am Geriatr Soc 2004;52:1863-1869.

4 Seidel UK, Gronewold J, Volsek M, et al: The prevalence, severity, and association with $\mathrm{HbAlc}$ and fibrinogen of cognitive impairment in chronic kidney disease. Kidney Int 2014;85:693-702.

5 Sehgal AR, Grey SF, DeOreo PB, Whitehouse PJ: Prevalence, recognition, and implications of mental impairment among hemodialysis patients. Am J Kidney Dis 1997;30:41-49.

6 Griva K, Stygall J, Hankins M et al: Cognitive impairment and 7-year mortality in dialysis patients. Am J Kidney Dis 2010;56:693-703.

7 Drew DA, Weiner DE, Tighiouart H, et al: Cognitive function and all-cause mortality in maintenance hemodialysis patients. Am J Kidney Dis 2015;65:303-311.

8 Kurella Tamura M, Larive B, Unruh ML, et al: Prevalence and correlates of cognitive impairment in hemodialysis patients: the Frequent Hemodialysis Network trials. Clin J Am Soc Nephrol 2010;5:1429-1438.

9 Thornton WL, Shapiro RJ, Deria S, et al: Differential impact of age on verbal memory and executive functioning in chronic kidney disease. J Int Neuropsychol Soc 2007;13:344353.

10 Krishnan AV, Kiernan MC: Neurological complications of chronic kidney disease. Nat Rev Neurol 2009;5:542-551.

11 Kramer L, Madl C, Stockenhuber F, et al: Beneficial effect of renal transplantation on cognitive brain function. Kidney Int 1996;49: 833-838.

12 Griva K, Hansraj S, Thompson D, et al: Neuropsychological performance after kidney transplantation: a comparison between trans- plant types and in relation to dialysis and normative data. Nephrol Dial Transplant 2004; 19:1866-1874.

13 Griva K, Thompson D, Jayasena D, et al: Cognitive functioning pre- to post-kidney transplantation - a prospective study. Nephrol Dial Transplant 2006;21:3275-3282.

14 Harciarek M, Biedunkiewicz B, Lichodziejewska-Niemierko $M$, et al: Cognitive performance before and after kidney transplantation: a prospective controlled study of adequately dialyzed patients with end-stage renal disease. J Int Neuropsychol Soc 2009; 15:684-694.

15 Harciarek M, Biedunkiewicz B, Lichodziejewska-Niemierko M, et al: Continuous cognitive improvement 1 year following successful kidney transplant. Kidney Int 2011;79:13531360.

16 Radic J, Ljutic D, Radic M, et al: Kidney transplantation improves cognitive and psychomotor functions in adult hemodialysis patients. Am J Nephrol 2011;34:399-406.

17 Unruh ML, Sanders MH, Redline S, et al: Sleep apnea in patients on conventional thrice-weekly hemodialysis: comparison with matched controls from the Sleep Heart Health Study. J Am Soc Nephrol 2006;17:3503-3509.

18 R Core Team: R: a language and environment for statistical computing. Vienna, R Foundation for Statistical Computing, 2014. URL http://www.R-project.org/.

19 Wickham H, Francois R: dplyr: A Grammar of Data Manipulation. $\mathrm{R}$ package version 0.4.1. 2015. http://CRAN.R-project.org/ package $=$ dplyr.

20 Subirana I, Sanz H, Vila J: Building bivariate tables: the compareGroups package for R. J Stat Softw. 2014;57:1-16..

21 Wickham H: ggplot2: Elegant Graphics for Data Analysis. New York, Springer, 2009.

22 Therneau T: A Package for Survival Analysis in S. version 2.38. 2015. http:// CRAN.R-project.org/package $=$ survival.

23 McIntyre CW, Goldsmith DJ: Ischemic brain injury in hemodialysis patients: which is more dangerous, hypertension or intradialytic hypotension? Kidney Int 2015;87:1109-1115.
24 Gelb S, Shapiro RJ, Hill A, Thornton WL: Cognitive outcome following kidney transplantation. Nephrol Dial Transplant 2008;23: 1032-1038.

25 Kurella Tamura M, Xie D, Yaffe K, et al: Vascular risk factors and cognitive impairment in chronic kidney disease: the Chronic Renal Insufficiency Cohort (CRIC) study. Clin J Am Soc Nephrol 2011;6:248-256.

26 Seliger SL, Sarnak MJ: Subclinical vascular disease of the brain in dialysis patients. Am J Kidney Dis 2007;50:8-10.

27 Weiner DE, Scott TM, Giang LM, et al: Cardiovascular disease and cognitive function in maintenance hemodialysis patients. Am J Kidney Dis 2011;58:773-781.

28 Drew DA, Bhadelia R, Tighiouart $\mathrm{H}$, et al: Anatomic brain disease in hemodialysis patients: a cross-sectional study. Am J Kidney Dis 2013;61:271-278.

29 Madero M, Sarnak MJ: Does hemodialysis hurt the brain? Semin Dial 2011;24:266-268.

30 Lass P, Buscombe JR, Harber M, et al: Cognitive impairment in patients with renal failure is associated with multiple-infarct dementia. Clin Nucl Med 1999;24:561-565.

31 Morosanu AI, Alexa ID, Badescu M, Ilie AC [Correlation between cognitive impairment and cardiovascular risk factors in dialysis vs. non-dialysis elderly patients]. Rev Med Chir Soc Med Nat Iasi 2011;115:1057-1061.

32 Naganuma T, Uchida J, Tsuchida K, et al: Silent cerebral infarction predicts vascular events in hemodialysis patients. Kidney Int 2005;67:2434-2439.

33 Nakatani T, Naganuma T, Uchida J, et al: Silent cerebral infarction in hemodialysis patients. Am J Nephrol 2003;23:86-90.

34 Agganis BT, Weiner DE, Giang LM, et al: Depression and cognitive function in maintenance hemodialysis patients. Am J Kidney Dis 2010;56:704-712.

35 Kaya Y, Ozturkeri OA, Benli US, Colak T: Evaluation of the cognitive functions in patients with chronic renal failure before and after renal transplantation. Acta Neurol Belg 2013;113:147-155. 